\title{
Efficiency of Edible Films Containing Bioactive Antimicrobials on Refrigerated Pork Meat
}

\author{
Ramona Andreea DOCHIŢOIU, Dan Cristian VODNAR, Carmen SOCACIU \\ University of Agricultural Sciences and Veterinary Medicine, Food Science and Tehcnology \\ Department, Mănăștur Nr. 3-5, Cluj-Napoca, 400372 Romania; \\ *vodnardan@yahoo.co.uk
}

Bulletin UASVM Food Science and Technology 71(1) / 2014

ISSN-L 2344-2344; Print ISSN 2344-2344; Electronic ISSN 2344-5300

\begin{abstract}
Preserving food is an important link in ensuring their quality; it is always a matter of concern for consumers. Some of the most common microorganisms existing on the surface of refrigerated meat are the Escherichia coli and Listeria monocytogenes. The use of bioactive compounds from psyllium and noni juice could be a way for inactivation of unwanted bacteria and extend the shelf life of chilled meat due to antioxidant and antimicrobial properties of theirs bioactive compounds. The biofilm formed by fusing powdered psyllium with noni juice was tested on the inhibition of microorganisms on chilled meat for 37 days. The HPLC characterization of noni juice showed the presence of ascorbic acid in concentration of $84 \mathrm{mg} \%$. Data from this study would provide new formulation options for developing antimicrobial packaging films using tea extracts to improve the microbiological safety and quality of pork meat during refrigerated storage.
\end{abstract}

Keywords: biofilm, bioactive pack, Escherichia coli, Lysteria monocytogenes, chilled meat

\section{INTRODUCTION}

The current global market has experienced an increasing consumer demand for better quality of life, meeting the criteria of high quality and safety of food. This has lead companies and researchers to explore different ways to improve their productivity in terms of maintaining quality, freshness and food safety, such as using sustainable materials in food packaging (Mahalik and Nambiar 2010; Lin and Zhao, 2007; Vodnar 2012). There has been a growing interest in recent years to develop materials with film-forming capacity and having antimicrobial properties which help to improve health safety and shelf life (Arvanitoyannis and Stratakos, 2012; Shen et al., 2010; Tajkarimi et al., 2010).

The innovative products consisting of an active biofilm embedding bioactive compounds meant to help producers who have always been concerned to satisfy a wide range of consumers. So the willingness to eat always fresh products, easy to prepare and to keep better sensory properties, burned this idea that improves shelf life, organoleptic properties and food safety of meat products as "ready to eat" products (Trinetta et al., 2010; Vodnar, 2012).

The overall objective of this study was to develop effective antimicrobial films incorporating bioactive compounds from psyllium and noni juice for controlling L.monocytogenes and E.coli packaged pork meat. The effectiveness of these antimicrobial films was evaluated at refrigerated temperature $\left(4^{\circ} \mathrm{C}\right)$ for 37 days.

\section{MATERIALS AND METHODS}

For the selective isolation and cultivation of the species Escherichia coli and Listeria monocytogenes were used $12.6 \mathrm{~g}$ powder and $9.61 \mathrm{~g}$ powder Levine, Oxford to give $350 \mathrm{ml}$ and $250 \mathrm{ml}$ culture medium. Was used $3.75 \mathrm{~g}$ agar (nutrient broth) to obtain a $150 \mathrm{ml}$ culture medium for the development of each bacteria in hand that we will later use to infect controlled meat. 
Afterwards the media were autoclaved at $121^{\circ} \mathrm{C}$ for 15 minutes. The dilutions were made in sterile saline solution $0.9 \%$.

Noni juice characterization was done by the HPLC method of separating, identifying and dispensing of ascorbic acid to determine its antioxidant potential and we used an Agilent 1200 HPLC system with UV-Vis detector.

Separation was done on an Eclipse XDB-C18 column size $150 \times 4,6 \mathrm{~mm}$ with $5 \mu \mathrm{m}$ particle size.

The column was eluted under isocratic mobile phase system: water / acetonitile / Ac formic $94 / 5 / 1(\mathrm{v} / \mathrm{v} / \mathrm{v})$ with a flow rate of $0.5 \mathrm{ml} / \mathrm{min}$. The chromatograms were recorded at $240 \mathrm{~nm}$.

For the identification and determination of ascorbic acid in the sample was used a standard L-ascorbic acid, purity 99\% manufactured by Sigma.

Sample preparation was carried out once and for all during the running time of experiment, which is considered to be day 0 , which lasted until day 37,5 is performed in 7 rounds of 7 days and at $0,7,14,21$ and 37 days.

In order to perform the experiment, were used 20 pieces of meat (pork chop) approximately of 30 $\mathrm{g}$ each having a thickness of $1 \mathrm{~cm}$ by 4 pieces for each stage of the two for E. coli and two for Listeria monocytogenes.

The active biofilm was prepared by fusing powdered psyllium representing $3.7 \%$ to $22.3 \%$ . Noni juice over the double distilled water was added at a rate of $74 \%$, required 5 pieces of meat infected with $E$. coli controlled. The process was repeated for the other five pieces of meat infected with Listeria monocytogenes.

After completion of this step, the sample trays are maintained at refrigerator temperature between $0^{\circ} \mathrm{C}$ and $4^{\circ}$ the meat will monitor the integrity, appearance, color and odor throughout the experiment, namely at $0,7,14,21$ and 37 days .

Samples were taken after each step properly and realized as 8 dilutions, after which they were incubated in the appropriate culture media.

\section{RESULTS AND DISCUSSIONS}

Biofilm was initially liquid consistency, this being an important factor for facilitating uniform immersion, but because the gelling property of the web that psyllium has been made in a very short time (within 1 minute) requiring a high-speed browsing stage (Fig. 1).

At the surface of the meat biofilm appeared as a semitransparent gelatinous film with reddish colored influenced noni juice.

In order to determine the amount of ascorbic acid in the sample to a calibration curve made with ascorbic acid Sigma 99\% purity. 5 concentrations were prepared by dissolving the standards in distillated water. The concentrations of the standard and the peaks corresponding to the areas are given in the following table:

The ascorbic acid content in noni juice sample was registered as $83 \mathrm{mg} \%$.

The inhibitory effect of the active biofilm on the chilled meat infected controlled E. coli (Fig. 4) and Listeria monocytogenes (Fig. 5) in the experiment

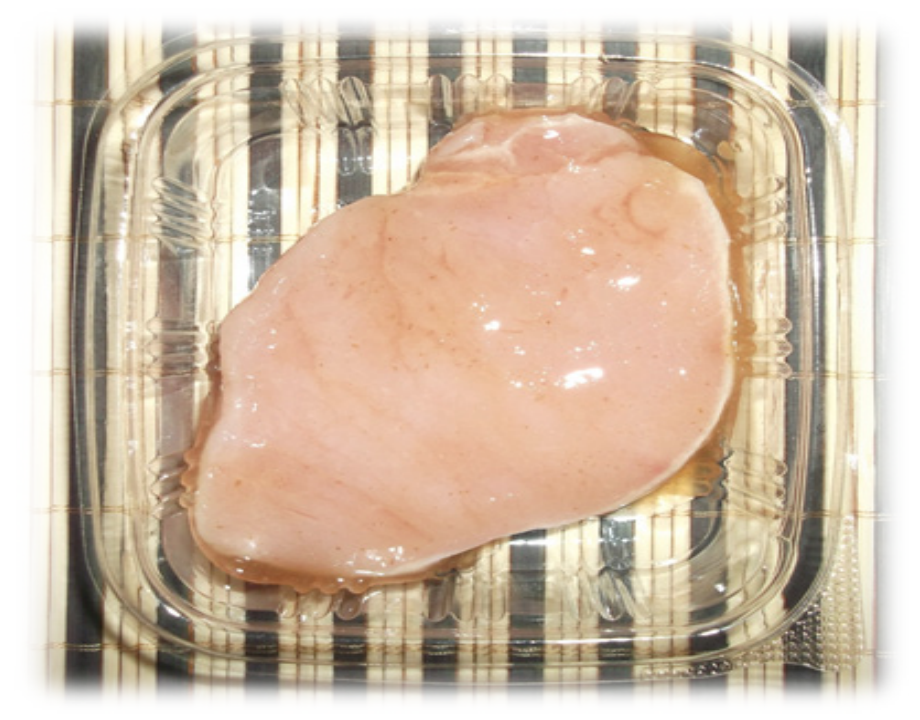

Fig. 1. Applied biofilm on the meat surface 


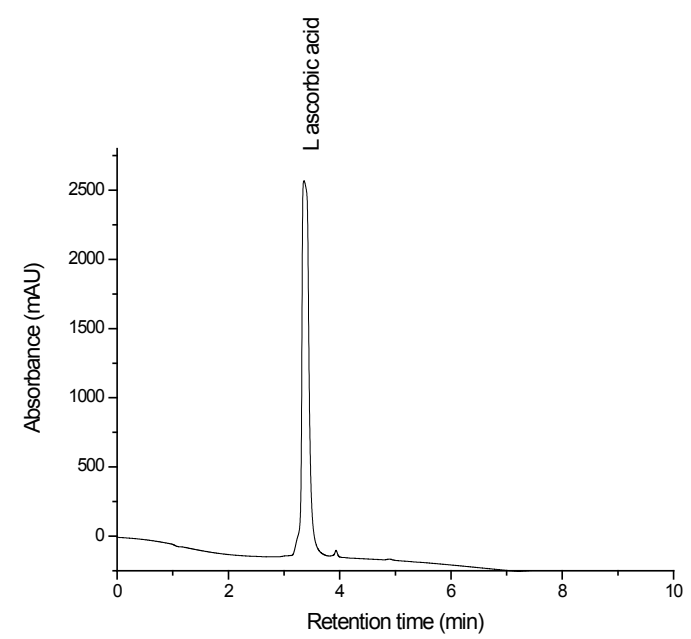

Fig. 2. Chromatogram of ascorbic acid $t_{R}=3,2 \min ($ standard)

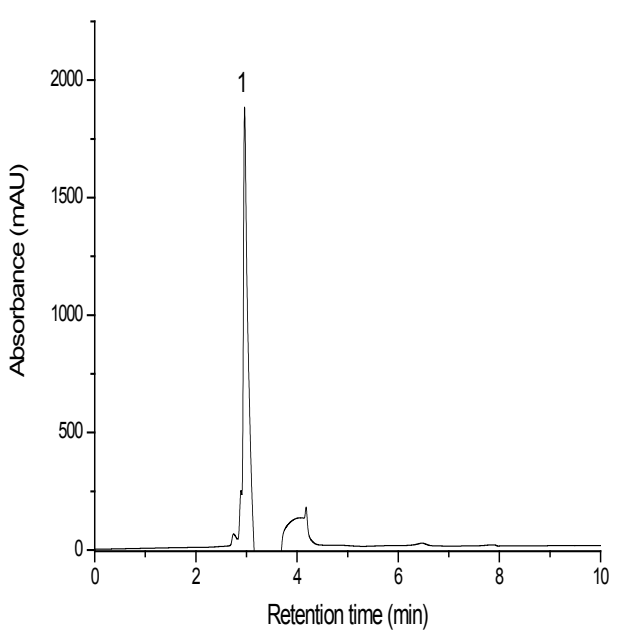

Fig. 3. Ascorbic acid identification in noni juice $\left(t_{R}=3,2 \mathrm{~min}\right)$

Table 1. Concentrations of ascorbic acid and associate area peaks values

\begin{tabular}{ccc}
\hline No. item & L-ascorbic acid concentration $(\mathbf{m g} / \mathbf{m l})$ & Area $(\mathbf{m A U})$ \\
\hline 1 & 0,8 & 40182 \\
\hline 2 & 0,7 & 34543 \\
\hline 3 & 0,6 & 28367 \\
\hline 4 & 0,5 & 22561 \\
\hline 5 & 0,2 & 8781 \\
\hline
\end{tabular}

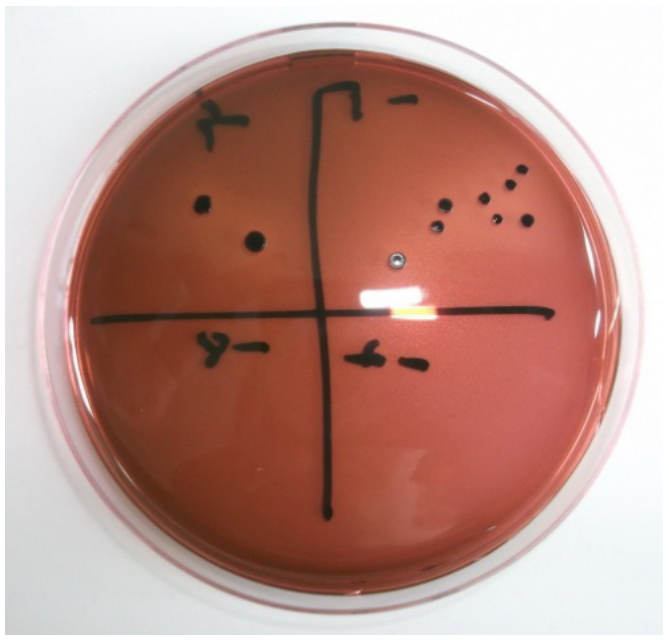

Fig. 4. Specific colonies of E.coli

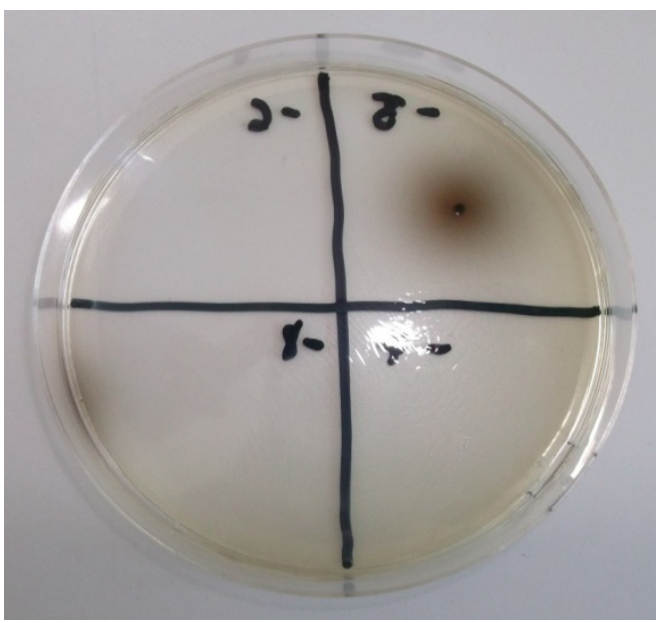

Fig. 5. Specific colonies of L. monocytogenes 
by monitoring the process and quantifying the microorganisms gradually took place in the 5 stages, namely at $0,7,14,9: 37$ p.m. as described in the previous section.

This has been made possible by considering each stage in the Petri dishes were removed after the incubation.

The image below illustrates an example of meat infected control sample containing only bacteria being Escherichia coli colonies grown on culture medium Levine in Eb sample from $10^{-1}$ to $10{ }^{8}$ dilution at day 0 .

After applying the active biofilm on the surface of infected meat with E. coli controlled, was found to reduce the number of bacteria in one logarithm (Figure 6). Active compounds inhibited the growth of $E$. coli in the sample (active biofilm) as opposed to blank (containing only bacteria) which was an increase of 1.5 logarithm E.coli bacteria. (Figure 6). After 7 days of storage, after the first step has found that the sample showed a regression in the number of decimal logarithm 0.5 E. coli compared to the control sample.

After applying the active biofilm on the surface of infected meat controlled L. monocytogenes was found to reduce the number of bacteria by 1.5 logarithm (Fig. 6). Active compounds inhibited the growth of $L$. monocytogenes in the sample (active biofilm) as opposed to blank (containing only bacteria) which was an increase of $L$. monocytogenes by 1.8 logarithm.

Our previous study combined (Vodnar 2012) the properties of a chitosan film with green and black tea extracts. Incorporation of tea extracts into the chitosan films considerably enhanced the effectiveness against L. monocytogenes ATCC 19115 on ham steak. Green tea (4\%) incorporated into chitosan-coated plastic film had a better antilisterial effect than green tea $2 \%$ or black tea $2 \%$ and $4 \%$. Data from study provided new formulation options for developing antimicrobial packaging films using tea extracts in order to improve the microbiological safety and quality of ham steak during room and refrigerated storage (Vodnar 2012).

Gómes- Estaca et al. (2010) reported that gelatin-chitosan film incorporating clove essential oils reduced drastically the population of gram negative bacteria on cod fish during chilled storage. More, Gómes- Estaca et al. (2007) reported that the stability of cold-smoked sardine muscle was improved by coating the muscle with functional gelatin film.

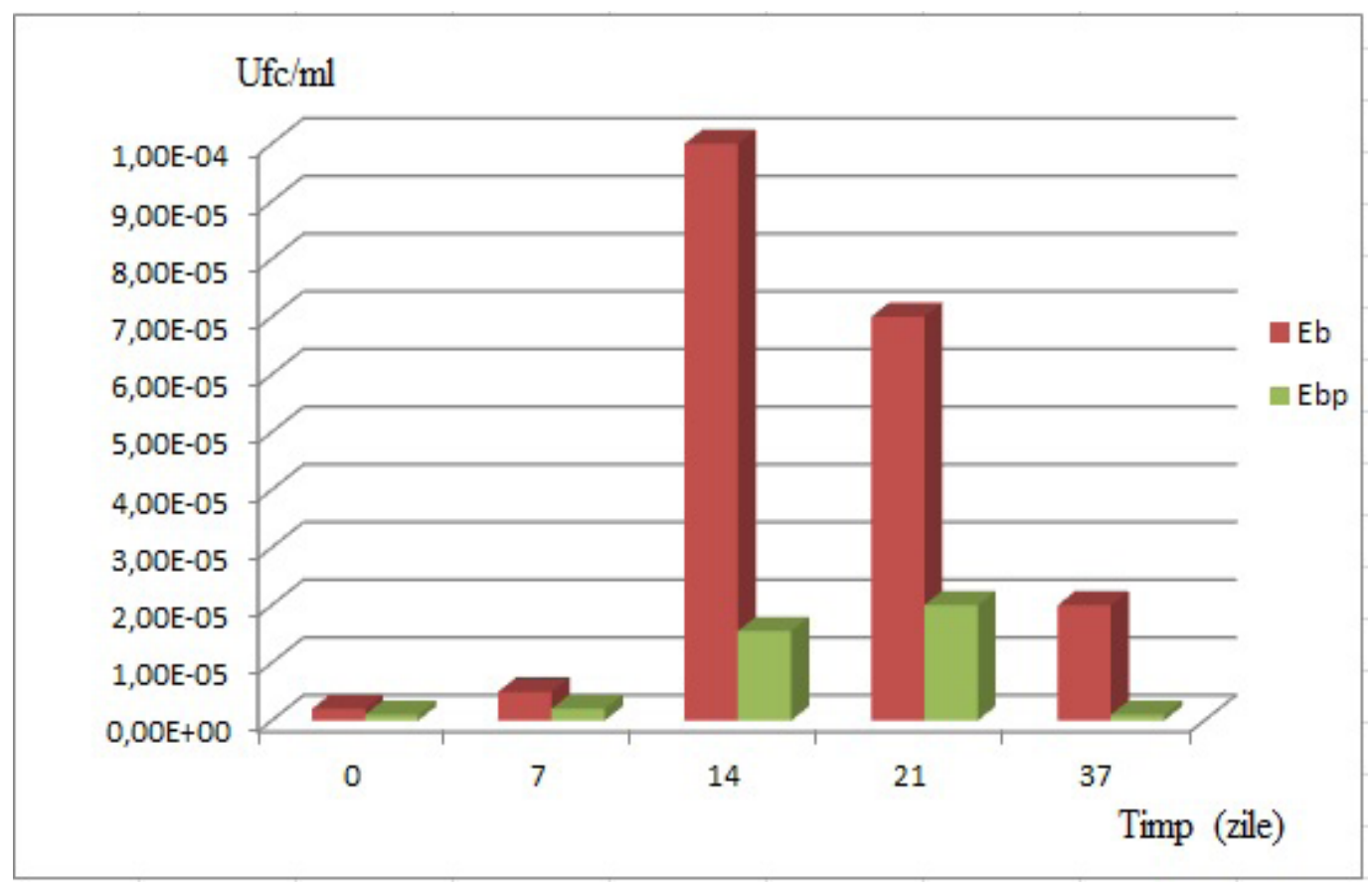

Fig. 6. The evolution of $E$. coli at $0,7,14,21$ and 37 days 


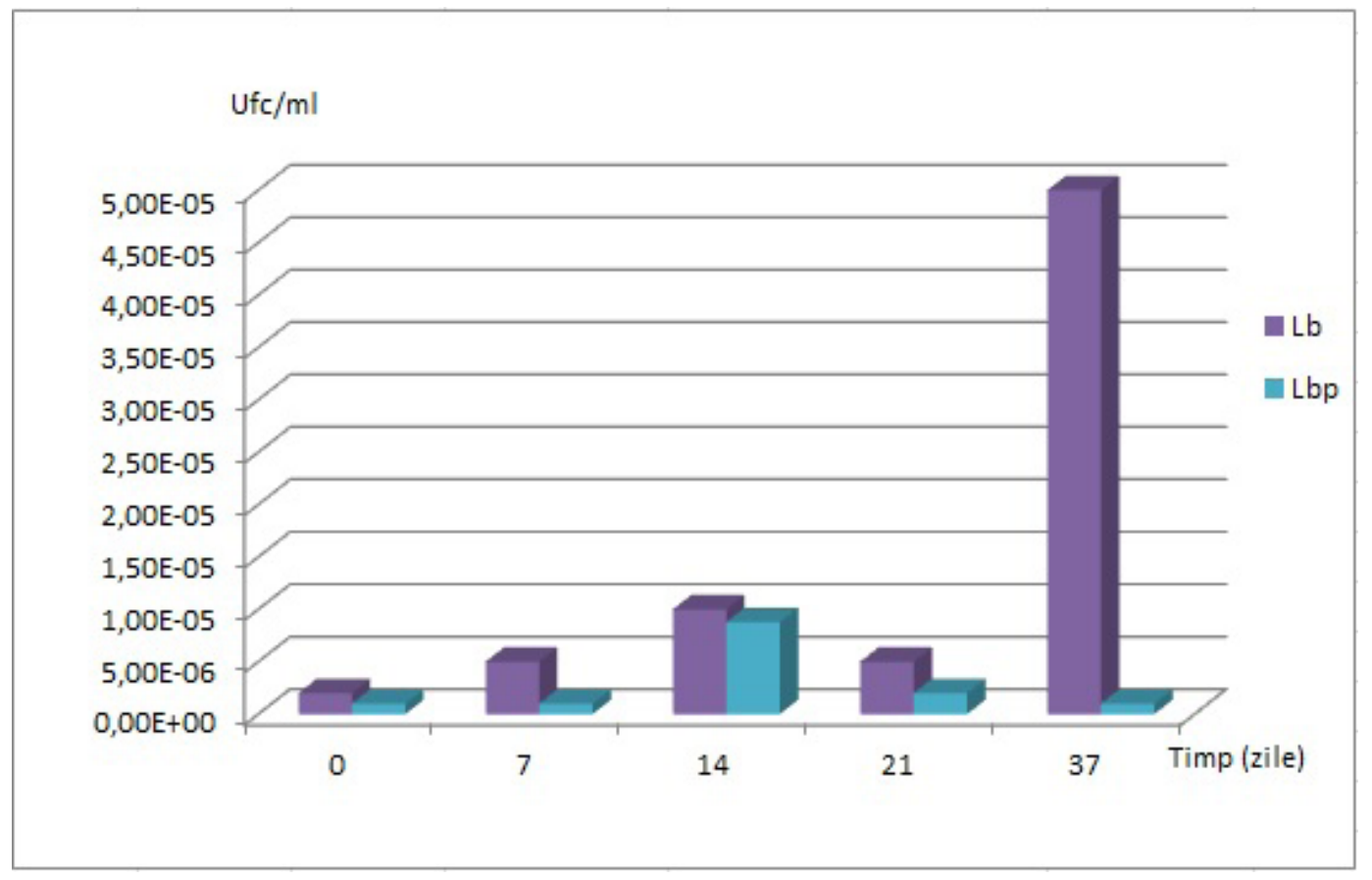

Fig. 7. The evolution of L. monocytogenes at $0,7,14,21$ and 37 days $\backslash$

\section{CONCLUSION}

As the chilled meat is an environment growth of microorganisms on the surface accidentally reached them, we proposed the use of innovative methods to address this shortcoming.

The antimicrobial effect of biofilm reduced the growth of Escherichia coli to 1 decimal logarithm, and Listeria monocytogenes growth was reduced to 1.5 logarithm during the five stages of the experiment. The antimicrobial effect exerted on the surface of the product (in which the pathogenic bacteria can grow ) can be an alternative to the addition of substances into the food matrix. Controlled release of antimicrobial substances in packaging type film, on the surface of food, over a period of time could be more advantageous than the incorporation of antimicrobial substances in the product.

\section{REFERENCES}

1. Arvanitoyannis, I.S. and Stratakos, A.Ch. (2012). Application of modified atmosphere packaging and active/smart technologies to red meat and poultry: A review, Food Bio Tech., 1423-1446.

2. Gómez-Estaca, J. et al. (2010). Biodegradable gelatinechitosan films incorporated with essential oils as antimi- crobial agents for fish preservation, Food Microb., 27:889896.

3. Gómez-Estaca, J. et al. (2007). Effect of functional edible films and high pressure processing on microbial and oxidative spoilage in cold-smoked sardine (Sardina pilchardus), Food Chem., 105:511-520.

4. Lin, D. and Zhao, Y. (2007). Innovations in the development and application of edible coatings for fresh and minimally processed fruits and vegetables, Compr Rev Food Sci Food Saf, 6:60-75.

5. Mahalik, N.P. and Nambiar, A.N. (2010). Trends in food packaging and manufacturing systems and technology, Trends Food Sci Tech., 21: 117-128.

6. Shen, X.L. et al. (2010). Antimicrobial and physical properties of sweet potato starch films incorporated with potassium sorbate or chitosan, Food Hydrocolloid, 24:285-290.

7. Tajkarimi, M.M. et al. (2010). Antimicrobial herb and spice compounds in food, Food Control, 21:1199-1218.

8. Trinetta, V. et al. (2010). A-containing pullulan film: an active packaging system to control epidemic clones of Listeria monocytogenes in ready-to-eat foods, J Food Safety, 30:366-381.

9. Vodnar, D.C. (2012). Inhibition of Listeria monocytogenes ATCC 19115 on ham steak by tea bioactive compounds incorporated into chitosan-coated plastic films, Chem Cent J, 6:74. 